\title{
Decomposição e liberação de nutrientes de soja cortada em diferentes estádios de desenvolvimento
}

\author{
Milton Parron Padovan(1), Dejair Lopes de Almeida( ${ }^{(2)}$, José Guilherme Marinho Guerra(2), \\ Raul de Lucena Duarte Ribeiro( ${ }^{(3)}$, Fábio Luiz de Oliveira $^{(4)}$, Leandro Azevedo Santos ${ }^{(3)}$, \\ Bruno José Rodrigues Alves ${ }^{(2)}$ e Sebastião Manhães Souto ${ }^{(2)}$
}

\begin{abstract}
(1)Universidade para o Desenvolvimento do Estado e da Região do Pantanal, Av. Presidente Vargas, no 1.775, CEP 79800-000 Dourados, MS. E-mail: Padovan@mail.uniderp.br (2)Embrapa Agrobiologia, BR 465, Km 7, CEP 23851-970 Seropédica, RJ. E-mail: dejair@cnpab.embrapa.br, gmguerra@cnpab.embrapa.br, bruno@cnpab.embrapa.br, smsouto@cnpab.embrapa.br (3)Universidade Federal Rural do Rio de Janeiro, Dep. de Agronomia, BR 465, Km 7, CEP 23851-970 Seropédica, RJ. E-mail: lucena@ufrrj.br, leoazevedo2001@yahoo.com.br (4)Universidade de Tocantins, Quadra 108 Sul, Alameda 11, Lote 03, Caixa Postal 173, CEP 77020-122 Palmas, TO. E-mail: fabioluiz@unitins.br
\end{abstract}

\begin{abstract}
Resumo - O objetivo deste trabalho foi avaliar a dinâmica de decomposição e a liberação de nutrientes da parte aérea de plantas de soja cultivar Celeste, para fins de adubação verde. Utilizou-se o delineamento experimental de blocos ao acaso com cinco repetições. Nas parcelas, as plantas foram cortadas rente ao solo aos 55, 85 e 115 dias após a emergência (DAE) e foram analisadas quanto à decomposição do material vegetal e liberação de N, P, K, Ca e Mg, colocando-se 60 g de matéria fresca da parte aérea em sacos de tela, distribuídos sobre a superfície das parcelas no campo. Nas subparcelas, as taxas de redução de massa de matéria seca e de liberação de nutrientes foram monitoradas mediante análises aos 5, 10, 15, 30, 60, 90, 120 e 150 dias após os cortes das plantas. O modelo exponencial $\mathrm{X}=\mathrm{X}_{0} \mathrm{e}^{-\mathrm{kt}}$ demonstrou dinâmicas não muito contrastantes, para os materiais cortados aos 55, $85 \mathrm{e}$ 115 DAE. Apesar de o clima possivelmente ter influenciado na liberação de K, não causou diferenças significativas na decomposição de matéria seca e na liberação de $\mathrm{N}$ e fósforo. As plantas de soja, cortadas em diferentes estádios de desenvolvimento, apresentam taxas similares de decomposição e de liberação de nutrientes.
\end{abstract}

Termos para indexação: Glycine max, dinâmica de decomposição, tempo de meia-vida, agricultura orgânica.

\section{Decomposition and nutrient release from soybean cut at different growth stages}

\begin{abstract}
The objective of this work was to evaluate the dynamics of decomposition and release of nutrients from aerial plant parts of the soybean cultivar Celeste used as green manure. A randomized block design was adopted with five replications. Soybean plants were cut close to the soil surface 55, 85 and 115 days after emergency (DAE), and analysed to determine their decomposition and release of N, P, K, Ca and Mg, by placing $60 \mathrm{~g}$ of fresh material of the aerial part in nylon screen bags, that were distributed on field plots surface. The rates of loss of dry matter and of nutrient release were monitored in the 5, 10, 15, 30, 60, 90, 120 and 150 days after plant cut. The exponential model $\mathrm{X}=\mathrm{X}_{0} \mathrm{e}^{-\mathrm{kt}}$ demonstrated that the dynamics of the material cut in the 55, 85 and $115 \mathrm{DAE}$ were not so contrasting. The climate could have interfered on the results, principally on K release rates, however it did not cause mean differences on the biomass decomposition and $\mathrm{N}$ and $\mathrm{P}$ release. Soybean plant material from cut plants, at different growth stages, present similar rates of decomposition and nutrient release.
\end{abstract}

Index terms: Glycine max, decomposition dynamics, half-life time, organic agriculture.

\section{Introdução}

Em sistemas de produção sob manejo orgânico, onde não é permitida a adubação com produtos industrializados, o uso de leguminosas como fonte de $\mathrm{N}$ para culturas de interesse comercial e alimentar é muito importante, principalmente nas condições em que a aplicação de esterco pode ser economicamente proibitiva para o sistema de produção (Almeida, 1991; Oliveira, 2001).
Blevins et al. (1990), Holderbaum et al. (1990) e Opkava et al. (2003) destacam que além de substituírem os adubos nitrogenados, as leguminosas permitem o aumento do reservatório de $\mathrm{N}$ no solo, melhorando sua fertilidade. Além disso, elas absorvem nutrientes das camadas subsuperficiais do solo e os liberam, posteriormente, na camada superficial, mediante a decomposição dos resíduos vegetais (Calegari et al., 1993; Duda et al., 2003), exercendo importante papel na ciclagem de nutrientes. 
A escolha do adubo verde e os critérios de manejo dependem do sistema de produção em que estão inseridos, sendo importante que a decomposição e a liberação de nutrientes ocorra de forma sincronizada com a demanda das plantas que receberam a adubação (Calegari, 2002). Nesse contexto, o conhecimento sobre a decomposição de adubos verdes e a dinâmica de liberação de nutrientes é muito importante, especialmente quando utilizados em pré-cultivos ou consorciados com culturas de interesse alimentício e comercial. Entretanto, estudos dessa natureza ainda são escassos, no Brasil, destacando-se os trabalhos de Da Ros (1993), Resende (2000), Espíndola (2001) e Oliveira (2001). Quanto à soja, que possui um grande potencial como adubo verde (Padovan et al., 2002), há poucos registros na literatura sobre o assunto no País, como os de Dutra (1919), Scaranari \& Inforzato (1952) e Cardoso (1956).

O objetivo deste trabalho foi analisar a dinâmica de decomposição e a liberação de nutrientes pela parte aérea de plantas de soja, para fins de adubação verde.

\section{Material e Métodos}

Utilizou-se a cultivar de soja Celeste, pela sua elevada capacidade de acumulação de massa e nutrientes (Padovan, 2002).

O trabalho foi desenvolvido no ano agrícola 2000/2001, no Sistema Integrado de Produção Agroecológica (SIPA), em que o manejo segue os preceitos da agricultura orgânica desde 1993. A área experimental está localizada em Seropédica, RJ, a $22^{\circ} 46$ 'S, $43^{\circ} 41^{\prime} \mathrm{W}$ e $33 \mathrm{~m}$ de altitude. O solo é classificado como Argissolo Vermelho-Amarelo (Embrapa, 1999), e sua análise química $(0-20 \mathrm{~cm})$ apresentou pH (água) 6,4; $\mathrm{Al}^{3+}, 0 \mathrm{cmol}_{\mathrm{C}} \mathrm{dm}^{-3} ; \mathrm{Ca}^{2+}$, $3,4 \mathrm{cmol}_{\mathrm{C}} \mathrm{dm}^{-3} ; \mathrm{Mg}^{2+}, 1,9 \mathrm{cmol}_{\mathrm{C}} \mathrm{dm}^{-3} ; \mathrm{P}, 52 \mathrm{mg} \mathrm{dm}^{-3} ; \mathrm{e} \mathrm{K}^{+}$, $145 \mathrm{mg} \mathrm{dm}^{-3}$.

O delineamento adotado foi o de blocos ao acaso, em parcelas subdivididas, com três tratamentos (coleta da parte aérea das plantas aos 55, 85 e 115 dias após a emergência - DAE) representando as parcelas, e com cinco repetições. As parcelas foram constituídas por oito fileiras de $10 \mathrm{~m}$ de comprimento, espaçadas de $0,5 \mathrm{~m}$ entre si, com densidade de 12 a 20 plantas $\mathrm{m}^{-1}$. Aplicaram-se em toda área experimental, 0,2 $\mathrm{t} \mathrm{ha}^{-1}$ de termofosfato magnesiano e $0,2 \mathrm{t} \mathrm{ha}^{-1}$ de cinzas de lenha, como fontes de macronutrientes e micronutrientes.

As sementes receberam inóculos das estirpes BR 29 e BR 96 de Bradyrhizobium japonicum, e a semea- dura foi realizada em 2/11/2000, na forma de plantio direto, após o cultivo de milho consorciado com feijão.

O controle das plantas espontâneas, nos estádios iniciais de desenvolvimento da cultura, foi realizado mediante a capina manual aos 20 DAE da soja. A ocorrência de insetos-pragas foi monitorada a cada dois dias, e não foram constatados danos que justificassem qualquer intervenção para o seu controle.

Aos 55, 85 e 115 DAE, correspondentes aos estádios $\mathrm{R}_{1}$ (início de florescimento da soja), $\mathrm{R}_{3}$ (início de frutificação) e $R_{5} / R_{6}$ (entre início de formação da semente ou início da granação e a granação plena ou semente desenvolvida), respectivamente, 40 plantas $\mathrm{m}^{-2}$ foram cortadas rente ao solo e avaliadas quanto à biomassa e ao conteúdo de nutrientes acumulados. A massa de matéria seca acumulada nos cortes aos 55, 85 e 115 DAE foi, respectivamente, 5,7, 7,3 e 5,5 $\mathrm{Mg} \mathrm{ha}^{-1}$.

Foram colocados $60 \mathrm{~g}$ de material fresco da parte aérea de uma planta inteira, em sacolas de decomposição, com 25x25 cm e abertura de malha de $4 \mathrm{~mm}$, para possibilitar a entrada da mesofauna e macrofauna do solo, responsáveis pela trituração dos resíduos vegetais, facilitando a decomposição do material pelos microrganismos. A determinação da massa do material colocado nas sacolas de decomposição foi feita pela secagem de amostras em estufa a $65^{\circ} \mathrm{C}$, até alcançarem valor constante.

As sacolas de decomposição foram distribuídas sobre a superfície das parcelas do experimento no campo, em contato com o solo. A quantidade de matéria seca e de nutrientes no material remanescente, em cada sacola de decomposição, foi monitorada mediante coletas realizadas nas subparcelas aos $5,10,15,30,60,90,120$ e 150 dias após a instalação do ensaio no campo. Os resíduos vegetais coletados foram secados em estufa, à temperatura de $65^{\circ} \mathrm{C}$, até alcançarem massa constante, e, então, moídos. Para a análise de N, utilizou-se a digestão sulfúrica e destilação à vapor (Alves et al., 1994). Os conteúdos de $\mathrm{P}, \mathrm{K}$, Ca e Mg foram determinados a partir da digestão nitroperclórica, conforme Silva (1999).

A decomposição do resíduo vegetal da soja e a liberação de nutrientes foram estimadas pela variação da quantidade e conteúdo de N, P, K, Ca e Mg no material remanescente nas sacolas de decomposição. Esses valores foram ajustados ao modelo exponencial simples: $\mathrm{X}=\mathrm{X}_{0} \mathrm{e}^{-\mathrm{kt}}$, em que $\mathrm{X}$ é a quantidade de matéria seca ou nutriente remanescente, após um período de tempo t, em dias, e $\mathrm{X}_{0}$ é a quantidade de matéria seca ou nutriente, no início do ensaio. Para os resíduos vegetais das 
diferentes épocas de corte, foram calculados os tempos de meia-vida $\left(\mathrm{t}_{1 / 2}\right)$, utilizando-se a equação: $\mathrm{t}_{1 / 2}=\ln 2 / \mathrm{k}$, em que $\mathrm{k}$ é derivado de cada equação, obtida do ajuste do modelo aos dados de matéria seca e conteúdo de nutrientes encontrados no estudo de incubação.

Os resultados foram submetidos à análise de variância, e as médias comparadas pelo teste de Tukey a 5\% de probabilidade.

\section{Resultados e Discussão}

Diferenças significativas, entre os resíduos vegetais oriundos das épocas de corte da soja, foram constatadas apenas aos 15 dias para o $\mathrm{N}$ e $\mathrm{P}$, e aos 30 dias de incubação para o K, Ca e Mg remanescentes (Tabela 1). Quanto à decomposição do resíduo vegetal, houve dife- rença significativa somente na terceira época de corte (115 DAE), com 40\% de matéria seca remanescente aos 30 dias após o corte, enquanto a primeira e segunda épocas ainda apresentavam 51 e 56\% de massa, respectivamente (Tabela 1). Essa diferença pontual se diluiu com o ajuste do modelo aos dados obtidos de todas as datas de amostragem, o que se traduziu em tempos de meia-vida da massa da parte aérea da soja semelhantes para as três épocas (Tabela 2). O padrão de decomposição da matéria seca da soja, nos três estádios de desenvolvimento em que foi submetida ao corte, apresentou-se semelhante ao observado por Resende (2000), para diversas leguminosas, e apresentou duas fases distintas, sendo a primeira considerada rápida, até os 30 dias após o corte da soja, e a segunda, mais lenta, a partir deste período (Tabela 1).

Tabela 1. Biomassa (matéria seca) e conteúdo de nutrientes remanescentes nas plantas de soja 'Celeste’, produzidas sob manejo orgânico e cortadas em três épocas (55, 85 e 115 DAE), correspondentes a diferentes estádios de desenvolvimento, e analisadas em nove dias distintos, após o corte ${ }^{(1)}$.

\begin{tabular}{|c|c|c|c|c|c|c|}
\hline $\begin{array}{l}\text { Amostragem } \\
\text { (DAC) }^{(2)}\end{array}$ & $\begin{array}{c}1^{\text {a época }} \\
(55 \mathrm{DAE})^{(3)}\end{array}$ & $\begin{array}{c}2^{\mathrm{a}} \text { época } \\
\text { (85 DAE) }\end{array}$ & $\begin{array}{c}3^{\text {a época }} \\
\text { (115 DAE) }\end{array}$ & $\begin{array}{c}1 \text { a época } \\
\text { (55 DAE) }\end{array}$ & $\begin{array}{l}2 \text { a época } \\
\text { (85 DAE) }\end{array}$ & $\begin{array}{c}\text { 3a época } \\
\text { (115 DAE) }\end{array}$ \\
\hline & \multicolumn{3}{|c|}{----------- Biomassa remanescente (\%) ----------- } & \multicolumn{3}{|c|}{--otal remanescente (\%) ---- N- } \\
\hline 0 & $100 \mathrm{a}$ & $100 \mathrm{a}$ & $100 \mathrm{a}$ & $100 \mathrm{a}$ & $100 \mathrm{a}$ & $100 \mathrm{a}$ \\
\hline 5 & $91 \mathrm{a}$ & $90 \mathrm{a}$ & $87 \mathrm{a}$ & $96 a$ & $99 \mathrm{a}$ & $98 \mathrm{a}$ \\
\hline 10 & $77 \mathrm{a}$ & $83 a$ & $75 \mathrm{a}$ & $75 \mathrm{ab}$ & $97 \mathrm{a}$ & $66 b$ \\
\hline 15 & $68 \mathrm{a}$ & $74 a$ & $70 \mathrm{a}$ & $54 \mathrm{~b}$ & $81 \mathrm{a}$ & $59 \mathrm{ab}$ \\
\hline 30 & $51 \mathrm{a}$ & $56 a$ & $40 \mathrm{~b}$ & $34 \mathrm{a}$ & $48 \mathrm{a}$ & $26 \mathrm{a}$ \\
\hline 60 & $34 \mathrm{a}$ & $35 \mathrm{a}$ & $28 \mathrm{a}$ & $20 \mathrm{a}$ & $29 \mathrm{a}$ & $19 \mathrm{a}$ \\
\hline 90 & $27 \mathrm{a}$ & $24 a$ & $28 \mathrm{a}$ & $15 \mathrm{a}$ & $19 \mathrm{a}$ & $17 \mathrm{a}$ \\
\hline 120 & $26 a$ & $24 \mathrm{a}$ & $24 \mathrm{a}$ & $14 \mathrm{a}$ & $17 \mathrm{a}$ & $14 \mathrm{a}$ \\
\hline \multirow[t]{2}{*}{150} & $16 \mathrm{a}$ & $18 \mathrm{a}$ & $19 \mathrm{a}$ & $7 \mathrm{a}$ & $11 \mathrm{a}$ & $11 \mathrm{a}$ \\
\hline & \multicolumn{3}{|c|}{--------- P remanescente (\%) ------------- } & \multicolumn{3}{|c|}{------------- K remanescente (\%) ------------- } \\
\hline 0 & $100 \mathrm{a}$ & $100 \mathrm{a}$ & $100 \mathrm{a}$ & $100 \mathrm{a}$ & $100 \mathrm{a}$ & $100 \mathrm{a}$ \\
\hline 5 & $82 \mathrm{a}$ & $100 \mathrm{a}$ & $87 a$ & $55 \mathrm{~b}$ & $85 a$ & $87 a$ \\
\hline 10 & $63 b$ & $92 \mathrm{a}$ & $74 \mathrm{ab}$ & $38 \mathrm{c}$ & $73 a$ & $57 \mathrm{~b}$ \\
\hline 15 & $57 \mathrm{~b}$ & $88 \mathrm{a}$ & $71 \mathrm{ab}$ & $27 \mathrm{c}$ & $68 \mathrm{a}$ & $43 b$ \\
\hline 30 & $40 \mathrm{a}$ & $49 a$ & $28 \mathrm{a}$ & $8 b$ & $36 a$ & $6 \mathrm{~b}$ \\
\hline 60 & $22 \mathrm{a}$ & $26 a$ & $16 a$ & $4 a$ & $5 a$ & $5 \mathrm{a}$ \\
\hline 90 & $11 \mathrm{a}$ & $14 a$ & $15 \mathrm{a}$ & $2 \mathrm{a}$ & $2 \mathrm{a}$ & $2 a$ \\
\hline 120 & $8 \mathrm{a}$ & $11 \mathrm{a}$ & $7 a$ & 1a & $1 \mathrm{a}$ & $2 a$ \\
\hline \multirow[t]{2}{*}{150} & $3 a$ & $8 \mathrm{a}$ & $5 \mathrm{a}$ & $1 \mathrm{a}$ & $1 \mathrm{a}$ & $1 \mathrm{a}$ \\
\hline & \multicolumn{3}{|c|}{--------------- Ca remanescente (\%) -------------- } & \multicolumn{3}{|c|}{------------- Mg remanescente $(\%)$------------ } \\
\hline 0 & $100 \mathrm{a}$ & $100 \mathrm{a}$ & $100 \mathrm{a}$ & $100 \mathrm{a}$ & $100 \mathrm{a}$ & $100 \mathrm{a}$ \\
\hline 5 & $95 \mathrm{a}$ & $91 \mathrm{a}$ & $96 a$ & $84 a$ & $92 \mathrm{a}$ & $89 \mathrm{a}$ \\
\hline 10 & $80 \mathrm{a}$ & $90 \mathrm{a}$ & $91 \mathrm{a}$ & $69 b$ & $90 \mathrm{a}$ & $68 \mathrm{~b}$ \\
\hline 15 & $73 \mathrm{a}$ & $86 a$ & $82 \mathrm{a}$ & $61 \mathrm{~b}$ & $82 \mathrm{a}$ & $64 \mathrm{~b}$ \\
\hline 30 & $60 \mathrm{a}$ & $70 \mathrm{a}$ & $36 b$ & $42 b$ & $59 \mathrm{a}$ & $27 \mathrm{c}$ \\
\hline 60 & $35 \mathrm{a}$ & $29 \mathrm{a}$ & $26 \mathrm{a}$ & $24 \mathrm{a}$ & $23 \mathrm{a}$ & $15 \mathrm{a}$ \\
\hline 90 & $20 \mathrm{a}$ & $19 \mathrm{a}$ & $24 \mathrm{a}$ & $12 \mathrm{a}$ & $15 \mathrm{a}$ & $14 \mathrm{a}$ \\
\hline 120 & $14 a$ & $17 \mathrm{a}$ & $20 \mathrm{a}$ & $9 a$ & $14 \mathrm{a}$ & $10 \mathrm{a}$ \\
\hline 150 & $7 \mathrm{a}$ & $10 \mathrm{a}$ & $12 \mathrm{a}$ & $5 \mathrm{a}$ & $8 \mathrm{a}$ & $6 a$ \\
\hline
\end{tabular}

(1)Médias (cinco repetições) seguidas da mesma letra, na linha, não diferem entre si pelo teste de Tukey a 5\% de probabilidade; os coeficientes de variação (CV) para biomassa, N total, P, K, Ca e Mg remanescentes foram 10,30, 31,08, 31,17, 21,67, 20,36 e 17,99\%, respectivamente. (2)DAC: dias após o corte da soja. (3)DAE: dias após a emergência da soja. 
O padrão de mineralização do $\mathrm{N}$ foi mais rápido do que o da decomposição do resíduo vegetal da soja, tendo variado de 20 a 34 dias para que a metade da quantidade inicial fosse liberada (Tabela 2). Houve diferença significativa na liberação do $\mathrm{N}$ existente na biomassa, apenas entre a segunda e terceira épocas, e a primeira e segunda épocas, aos 10 e 15 dias depois do corte, respectivamente (Tabela 1 ).

Da Ros (1993) constatou que a diminuição das quantidades de matéria seca e de $\mathrm{N}$ de quatro leguminosas foi extremamente rápida na fase inicial da decomposição. Esse autor observou, também, que aos 30 dias depois do corte das espécies, apenas $62 \%$ da matéria seca e $40 \%$ da quantidade de $\mathrm{N}$ adicionado ainda permaneciam na superfície do solo. A taxa inicial alta de diminuição de matéria seca é atribuída à remoção da fração solúvel em água pela chuva, e à facilidade de

Tabela 2. Parâmetros da equação $X=\mathrm{X}_{0} \mathrm{e}^{-\mathrm{kt}}$ ajustada aos valores de decomposição da biomassa (matéria seca), liberação de nutrientes e tempo de meia vida, depois dos cortes realizados na soja aos 55, 85 e 115 dias após a emergência, em média de cinco repetições.

\begin{tabular}{|c|c|c|c|}
\hline 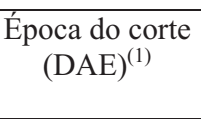 & $\begin{array}{c}\text { Constante de } \\
\text { decomposição k } \\
\left(\mathrm{g} \mathrm{g}^{-1} \mathrm{dia}^{-1}\right)\end{array}$ & $\begin{array}{l}\text { Tempo de meia } \\
\text { vida }\left(\mathrm{t}_{1 / 2}\right) \\
(\text { dias })\end{array}$ & $\begin{array}{l}\text { Coeficiente de } \\
\text { determinação } \\
\left(\mathrm{R}^{2}\right)\end{array}$ \\
\hline & \multicolumn{3}{|c|}{ Biomassa } \\
\hline 55 & 0,01471 & 47 & 0,947 \\
\hline 85 & 0,01470 & 47 & 0,947 \\
\hline 115 & 0,01613 & 43 & 0,868 \\
\hline & \multicolumn{3}{|c|}{ Nitrogênio } \\
\hline 55 & 0,02538 & 27 & 0,928 \\
\hline 85 & 0,02032 & 34 & 0,957 \\
\hline 115 & 0,03391 & 20 & 0,845 \\
\hline & \multicolumn{3}{|c|}{ Fósforo } \\
\hline 55 & 0,02763 & 25 & 0,989 \\
\hline 85 & 0,02171 & 32 & 0,968 \\
\hline 115 & 0,03124 & 22 & 0,945 \\
\hline \multicolumn{4}{|c|}{ Potássio } \\
\hline 55 & 0,09471 & 7 & 0,898 \\
\hline 85 & 0,03499 & 20 & 0,861 \\
\hline 115 & 0,06041 & 11 & 0,943 \\
\hline \multicolumn{4}{|c|}{ Cálcio } \\
\hline 55 & 0,01754 & 40 & 0,997 \\
\hline 85 & 0,01681 & 41 & 0,971 \\
\hline 115 & 0,02012 & 34 & 0,901 \\
\hline \multicolumn{4}{|c|}{ Magnésio } \\
\hline 55 & 0,02538 & 27 & 0,987 \\
\hline 85 & 0,02032 & 34 & 0,961 \\
\hline 115 & 0,03390 & 20 & 0,916 \\
\hline
\end{tabular}

(1)55 DAE: início do florescimento; 85 DAE: início da frutificação; 115 DAE: entre o início da formação da semente e o desenvolvimento pleno da semente. decomposição microbiana dessa fração, de baixa relação $\mathrm{C} / \mathrm{N}$, mesmo quando os resíduos culturais permanecem na superfície do solo.

O P apresentou liberação similar nas três épocas de corte, tendo diferido, significativamente, somente entre a primeira e segunda épocas, aos 10 e 15 dias depois dos respectivos cortes (Figura 1; Tabela 1). O tempo de meia-vida do $\mathrm{P}$ remanescente no resíduo vegetal da soja variou de 22 a 32 dias (Tabela 2).

Com relação ao K, foram observados tempos de meiavida entre 7 e 20 dias, para as diferentes épocas de corte, demonstrando a rápida velocidade de liberação desse nutriente, independentemente da época de corte (Tabela 2). No período de 5 a 30 dias depois do corte, constatou-se diferença significativa na porcentagem de K remanescente no resíduo vegetal da soja, entre os três estádios de desenvolvimento em que a leguminosa foi cortada (Tabela 1). Resultados semelhantes com esse nutriente foram obtidos por Resende (2000), Espíndola (2001) e Oliveira (2001), em estudos que envolveram diferentes espécies de leguminosas e ecorregiões no País. O padrão de rápida liberação do K, pelos resíduos das plantas, também foi verificado por Lupwayi \& Haque (1998), com as espécies arbóreas Sesbania sesban e Leucaena leucocephala, nas quais a maioria do $\mathrm{K}$ foi liberado na primeira semana depois do corte e incubação dos resíduos com o solo. A quase totalidade do K está presente sob a forma iônica nas plantas, o que facilita sua liberação. Segundo Taiz \& Zeiger (1991), a não participação do K na formação de estruturas orgânicas da planta pode causar a sua rápida liberação.

O Ca apresentou o maior tempo de meia-vida no resíduo vegetal da soja, entre os nutrientes estudados, e variou de 34 a 41 dias (Tabela 2), além de apresentar a menor variação na liberação, durante o processo de decomposição do resíduo vegetal, com diferença significativa da terceira época de corte, em relação à primeira e segunda épocas, apenas aos 30 dias depois dos corte (Tabela 1).

O processo de liberação de Mg, aos 10 e 15 dias depois do corte, apresentou diferença significativa na segunda época, com os maiores porcentuais remanescentes desse nutriente, em relação à primeira e terceira épocas. Aos 30 dias depois do corte, as três épocas diferiram significativamente entre si, na dinâmica de liberação desse elemento (Tabela 1). O tempo de meiavida do $\mathrm{Mg}$, no resíduo vegetal da soja, foi igual ao do $\mathrm{N}$ e semelhante ao $\mathrm{P}$, nas três épocas de corte (Tabela 2). 
Quando o experimento foi concebido, imaginava-se que o tempo de meia-vida dos resíduos e dos nutrientes $\mathrm{N}$, P, K, Ca e Mg, da parte aérea da soja, aumentasse à medida que a coleta da soja fosse realizada em estádios de desenvolvimento mais tardios, em decorrência da formação de tecidos mais recalcitrantes pela cultura, tal como observado para alfafa por Martiniello et al. (1997).

Fatores intrínsecos aos resíduos vegetais, como a sua composição bioquímica, principalmente o teor de lignina e a relação $\mathrm{C} / \mathrm{N}$, exercem um papel preponderante no processo de decomposição. Palm \& Sanchez (1991) enfatizam que a porcentagem de lignina, nos tecidos das plantas, influencia o processo de decomposição do resíduo vegetal e a liberação dos nutrientes, principalmente o N. Frankenberger \& Abdelmagid (1985) chamam a atenção às plantas com maiores teores de lignina, as quais apresentam decomposição mais lenta.

No entanto, neste trabalho, possíveis alterações qualitativas, decorrentes da fenologia da cultura da soja, não foram suficientes para alterar significativamente os padrões de decomposição. Aparentemente, houve influência climática no processo de liberação de nutrientes, especialmente do K, em razão da maior precipitação e melhor distribuição das chuvas, depois da terceira época de corte da soja, em relação à primeira e segunda épocas (Figura 1). Entretanto, pela fácil
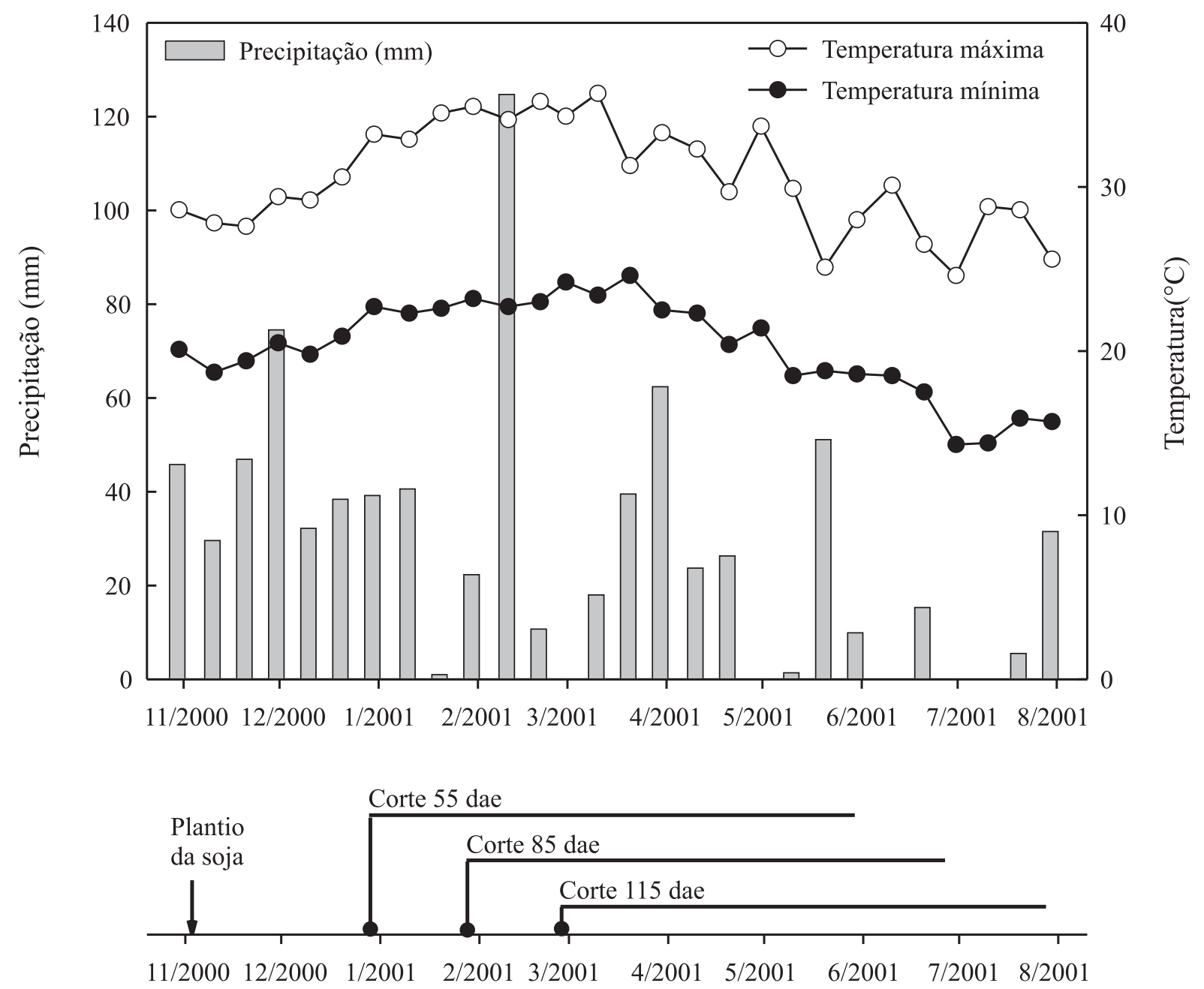

Período experimental

Figura 1. Precipitação pluvial acumulada a cada dez dias (mm) e temperaturas máximas e mínimas (médias de dez dias, em ${ }^{\circ} \mathrm{C}$ ), registradas durante o período de novembro de 2000 a agosto de 2001 (Posto Agrometeorológico da Estação Experimental da Pesagro de Itaguaí, RJ). No eixo adicional projetado do gráfico são identificados a data do plantio da soja e as datas dos cortes realizados aos 55, 85 e 115 dias após a emergência (dae), sendo o período de decomposição do material cortado demonstrado pelas linhas horizontais. 
mobilização do K no resíduo vegetal, as condições ambientais observadas no primeiro e segundo cortes não seriam limitantes para sua liberação, pois as alterações qualitativas, com a idade da cultura da soja, parecem não ser suficientes para mudar os padrões de decomposição de resíduos e liberação de nutrientes, especialmente o $\mathrm{N}$.

\section{Conclusão}

As plantas de soja cortadas em diferentes estádios de desenvolvimento apresentam semelhanças quanto às curvas de decomposição da biomassa e de liberação de N, P, Ca e Mg.

\section{Referências}

ALMEIDA, D.L. de. Contribuição da adubação orgânica para a fertilidade do solo. 1991. 192p. Tese (Mestrado) - Universidade Federal Rural do Rio de Janeiro, Seropédica.

ALVES, B.J.R.; SANTOS, J.C.F.; URQUIAGA, S.; BODDEY, M.R. Métodos de determinação do nitrogênio em solo e planta. In: ARAÚJO, R.S.; HUNGRIA, M. (Ed.). Manual de métodos empregados em estudos de microbiologia agrícola. Brasília: Embrapa-SPI, 1994. p.449-469.

BLEVINS, R.L.; HERBEK, J.H.; FRYE, W.W. Legume cover crops as a nitrogen source for no-till corn and grain sorghum. Agronomy Journal, v.82, p.769-772, 1990.

CALEGARI, A. Rotação de culturas e uso de plantas de cobertura. Agroecologia, v.2, p.14-17, 2002.

CALEGARI, A.; MONDARDO, A.; BULISANI, E.A.; WILDNER, L.P.; COSTA, M.B.B.; ALCÂNTARA, P.B.; MIYASAKA, S.; AMADO, T.J.C. Adubação verde no Sul do Brasil. 2.ed. Rio de Janeiro: AS-PTA, 1993. 346p.

CARDOSO, E.M. Contribuição para o estudo da adubação verde dos canaviais. 1956. 109p. Tese (Doutorado) - Escola Superior de Agricultura Luiz de Queiroz, Piracicaba.

DA ROS, C.O. Plantas de inverno para cobertura do solo e fornecimento de nitrogênio ao milho em plantio direto. 1993. 85p. Dissertação (Mestrado) - Universidade Federal de Santa Maria, Santa Maria.

DUDA, G.P.; GUERRA, J.G.M.; MONTEIRO, M.T.; DE-POLLI, H.; TEIXEIRA, M.G. Perennial herbaceous legumes as live soil mulches and their effects on $\mathrm{C}, \mathrm{N}$ and $\mathrm{P}$ of the microbial biomass. Scientia Agricola, v.60, p.139-147, 2003.

DUTRA, G.R.P. Adubos verdes: sua produção e modo de emprego. São Paulo: Secretaria da Agricultura Comércio e Obras Públicas do Estado de São Paulo, 1919. 76p.
EMBRAPA. Centro Nacional de Pesquisa de Solos (Rio de Janeiro, RJ). Sistema brasileiro de classificação de solos. Brasília: Embrapa-SPI; Rio de Janeiro: Embrapa-CNPS, 1999. 412 p.

ESPÍNDOLA, J.A.A. Avaliação de leguminosas herbáceas perenes usadas como cobertura viva do solo e sua influência sobre a produção da bananeira. 2001. 137p. Tese (Doutorado) Universidade Federal Rural do Rio de Janeiro, Seropédica.

FRANKENBERGER, W.T.; ABDELMAGID, H.M. Kinetic parameters of nitrogen mineralization rates of leguminous crops incorporated into soil. Plant and Soil, v.87, p.257-271, 1985.

HOLDERBAUM, J.F.; DECKER, A.M.; MEISINGER, J.J.; MULFORD, F.R.; VOUGH, L.R. Fall-seeded legume cover crops for no-tillage corn in the humid East. Agronomy Journal, v.82, p.117-124, 1990.

LUPWAYI, N.Z.; HAQUE, I. Mineralization of N, P, K, Ca and Mg from Sesbania and Leucaena leaves varying in chemical composition. Soil Biology and Biochemistry, v.30, p.337-343, 1998.

MARTINIELLO, P.; PAOLETTI, R.; BERARDO, N. Effect of phenological stages on dry matter and quality components in lucerne. European Journal of Agronomy, v.6, p.79-87, 1997.

OLIVEIRA, F.L. Manejo orgânico da cultura do repolho (Brassica oleracea var. capitata): adubação orgânica, adubação verde e consorciação. 2001. 87p. Dissertação (Mestrado) - Universidade Federal Rural do Rio de Janeiro, Seropédica.

OPKAVA, D.A.; NJOKU, J.C.; IKEORGU, J.E.G. Maiz response to green manure under the humid tropical conditions of South-Eastern Nigeria. Tropical Agriculture, v.80, p.1-5, 2003.

PADOVAN, M.P. Desempenho da soja, sob manejo orgânico, para produção de grãos e adubação verde. 2002. 88p. Tese (Doutorado) - Universidade Federal Rural do Rio de Janeiro, Seropédica.

PADOVAN, M.P.; ALMEIDA, D.L. de; GUERRA, J.G.M.; RIBEIRO, R. de L.D.; NDIAYE, A. Avaliação de cultivares de soja, sob manejo orgânico, para fins de adubação verde e produção de grãos. Pesquisa Agropecuária Brasileira, v.37, p.1705-1710, 2002.

PALM, C.A.; SANCHEZ, P.A. Nitrogen release from the leaves of some tropical legumes as affected by their lignin and polyphenolic contents. Soil Biology and Biochemistry, v.23, p.83-88, 1991.

RESENDE, A. A fixação biológica de nitrogênio (FBN) como suporte da produtividade e fertilidade nitrogenada dos solos na cultura da cana-de-açúcar: uso de adubos verdes. 2000. 124p. Dissertação (Mestrado) - Universidade Federal Rural do Rio de Janeiro, Seropédica.

SCARANARI, H.J.; INFORZATO, R. Sistema radicular das principais leguminosas empregadas como adubo verde em cafezal. Bragantia, v.2, p.291-297, 1952.

SILVA, F.C. da. Manual de análises químicas de solos, plantas e fertilizantes. Brasília: Embrapa-SNT, 1999. 370p.

TAIZ, L.; ZEIGER, E. Plant physiology. Redwood City: Benjamin/ Cummings Pub. Co., 1991. 559p.

Recebido em 25 de outubro de 2004 e aprovado em 18 de outubro de 2005 